\title{
First Asymmetric Cycloaddition of Carbonyl Ylides to Vinyl Sulfoxides and Furan-2(5H)-ones
}

\author{
José L. García Ruano, * Alberto Fraile, M. Rosario Martín, * and Alberto Núñez \\ Departamento de Química Orgánica, Universidad Autónoma de Madrid, Cantoblanco, 28049 Madrid, \\ Spain
}

joseluis.garcia.ruano@uam.es

\section{Supporting Information}

(28 pages)

General methods

S2

$\begin{array}{lll}\text { Experimental Procedures and characterization data of compounds } 5 \text { and } 7 & \text { S2-S5 }\end{array}$

${ }^{1} \mathrm{H}$ NMR spectrum of compound anti-5-exo $\quad$ S6

${ }^{13} \mathrm{C}$ NMR spectrum of compound anti-5-exo $\quad$ S7

${ }^{1} \mathrm{H}$ NMR spectrum of compound anti-5-endo $\quad$ S8

${ }^{13} \mathrm{C}$ NMR spectrum of compound anti-5-endo $\quad$ S9

$\begin{array}{lr}{ }^{1} \mathrm{H} \text { NMR spectrum of compound anti-6b-endo } & \mathrm{S} 10\end{array}$

${ }^{13} \mathrm{C}$ NMR spectrum of compound anti-6b-endo $\quad$ S11

$\begin{array}{lr}\text { COSY of compound anti-6b-endo } & \text { S12 }\end{array}$

$\begin{array}{lr}\text { NOESY of compound anti-6b-endo } & \text { S13 }\end{array}$

$\begin{array}{lr}\text { HMQC of compound anti-6b-endo } & \text { S14 }\end{array}$ 
$\begin{array}{lr}\text { COSY of compound anti-6b-exo } & \text { S15 }\end{array}$

$\begin{array}{lr}\text { NOESY of compound anti-6b-exo } & \text { S16 }\end{array}$

$\begin{array}{ll}\text { HMQC of compound anti-6b-exo } & \text { S17 }\end{array}$

$\begin{array}{ll}\text { COSY of compound syn-6b-exo } & \text { S18 }\end{array}$

$\begin{array}{ll}\text { NOESY of compound syn-6b-exo } & \text { S19 }\end{array}$

$\begin{array}{ll}\text { HMQC of compound syn-6b-exo } & \text { S20 }\end{array}$

$\begin{array}{ll}{ }^{1} \mathrm{H} \text { NMR spectra of compound anti-6a-endo } & \text { S21 }\end{array}$

${ }^{13} \mathrm{C}$ NMR spectra of compound anti-6a-endo $\quad$ S22

$\begin{array}{ll}\text { HMQC of compound anti-6a-endo } & \text { S23 }\end{array}$

$\begin{array}{ll}\text { COSY of compound anti-6a-exo } & \text { S24 }\end{array}$

$\begin{array}{ll}\text { NOESY of compound anti-6a-exo } & \text { S25 }\end{array}$

$\begin{array}{ll}\text { HMQC of compound anti-6a-exo } & \text { S26 }\end{array}$

$\begin{array}{ll}{ }^{1} \mathrm{H} \text { NMR spectrum of compound (+)-anti-7-exo } & \text { S27 }\end{array}$

$\begin{array}{ll}\text { HMQC of compound (-)-anti-7-exo } & \text { S28 }\end{array}$

General Methods. All reactions were performed in flame-dried glassware equipped with rubber septa under positive pressure of argon. Flash chromatography was carried out with silica gel 60 (230-400 mesh ASTM), and silica gel $\mathrm{F}_{254}$ plates were used for preparative TLC. NMR spectra were determined in $\mathrm{CDCl}_{3}$ solutions at 200 (or 300) and 50.3 (or 75) $\mathrm{MHz}$ for ${ }^{1} \mathrm{H}$ and ${ }^{13} \mathrm{C}-\mathrm{NMR}$, respectively. NMR parameters were assigned by HMQC, COSY and NOESY experiments.

\section{Cycloadditions of 2-benzopyrylium-4-olate to 5-methoxyfuran-2(5H)-one.}

A solution of $138 \mathrm{mg}(0.68 \mathrm{mmol})$ of $o$-methoxycarbonyl- $\alpha$-diazoacetophenone $(4)$ in THF (2.2 $\mathrm{mL})$ was added to a suspension of $\mathrm{Rh}_{2}(\mathrm{OAc})_{4}(7.5 \mathrm{mg}, 0.017 \mathrm{mmol})$ and furanone 3 (233 $\mathrm{mg}, 2.04$ $\mathrm{mmol}$ ), in $18 \mathrm{~mL}$ of the same solvent over a period of $2 \mathrm{~h}$ at room temperature. After thirty minutes once the addition of 4 to the solution of catalyst and 5-methoxyfuran-2(5H)-one $(3)$ was complete the solvent 
was removed in vacuo to give a 1:2 mixture of anti-5-exo/anti-5-endo. They are isolated by column chromatography (hexane-dichloromethane-diethyl ether, 4:2:1). The reported yields correspond to isolated products and are calculated from the amount of starting diazoketone 4 .

\section{1,4-Dimethoxy-3a,4,10,10a-tetrahydro-1 $H$-4,10-epoxybenzo[4,5]cyclohepta[1,2-c]furan-3,9-diones}

\section{(5).}

(anti-5-exo). 7\% Yield. Recrystallized from ether-hexane, mp 196-199 C. IR (KBr): 1799, 1774, 1700, 1601, 1172, 1127, 1121, 1046, 1019, 954. ${ }^{1} \mathrm{H}$ NMR $\delta: 8.03$ (d, 1H, J 7.7), $7.72(\mathrm{~m}, 1 \mathrm{H}), 7.56$ (m, 2H), $5.44(\mathrm{~d}, 1 \mathrm{H}, J$ 1.6), $4.90(\mathrm{~m}, 1 \mathrm{H}), 3.53(\mathrm{~s}, 3 \mathrm{H}), 3.52(\mathrm{~s}, 3 \mathrm{H}), 3.37(\mathrm{dd}, 1 \mathrm{H}, J 8.2$ and 0.6), 2.93 (ddd, 1H, $J$ 8.2, 1.6 and 1.0). ${ }^{13} \mathrm{C}$ NMR $\delta: 191.9,170.4$, and 143.1(C), 135.1, $129.8(\mathrm{CH}), 129.3(\mathrm{C}) 127.8,123.6$ $(\mathrm{CH}), 108.1(\mathrm{C}), 108.0$ and $84.5(\mathrm{CH}), 57.1$ and $54.2\left(\mathrm{CH}_{3}\right), 53.4$ and $49.0(\mathrm{CH})$.

(anti-5-endo). 10\% Yield. IR (film): 1780, 1703, 1601, 1297, 1120. ${ }^{1} \mathrm{H}$ NMR $\delta: 8.00$ (d., 1H, J 7.7 Hz), $7.68(\mathrm{~m}, 1 \mathrm{H}), 7.59(\mathrm{~d}, 1 \mathrm{H}, J$ 7.7), $7.54(\mathrm{~m}, 1 \mathrm{H}), 5.01(\mathrm{~d}, 1 \mathrm{H}, J$ 8.9), $4.95(\mathrm{~s}, 1 \mathrm{H}), 3.81(\mathrm{~d}, 1 \mathrm{H}, J 10.5)$,

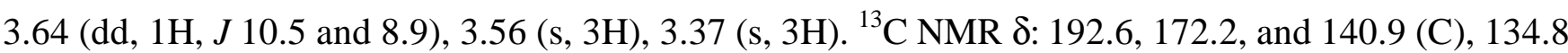
$(\mathrm{CH}), 130,0(\mathrm{C}), 129.8,126.7$ and $125.7(\mathrm{CH}), 108.1(\mathrm{C}), 102.7$ and $81.4(\mathrm{CH}), 56.8,52.9 ; 52.4$ and $48.5\left(\mathrm{CH}_{3}\right.$ and $\left.\mathrm{CH}\right)$.

\section{Desulfynilation of sulfinyl adducts 6}

\section{1-Ethoxy-4-methoxy-3a,4,10,10a-tetrahydro-1H-4,10-epoxybenzo[4,5]cyclohepta[1,2-c]furan-}

\section{3,9-diones (7):}

Activated Zinc. A $20 \mathrm{~g}$ portion of zinc dust in a fritted glass funnel was washed with $50 \mathrm{~mL}$ of $5 \%$ aqueous $\mathrm{HCl}$, water, and methanol, in that order. The "active" zinc was ground to remove lumps and then dried in vacuum.

The following experimental procedure is typical for the desulfinylation of sulfinyl adducts 6 :

To a stirred solution of $0.1 \mathrm{mmol}$ of syn-6b-exo in $7 \mathrm{~mL}$ of THF was added, at room temperature, 450 $\mathrm{mg}(6.9 \mathrm{mmol})$ of zinc and $7 \mathrm{~mL}$ of saturated aqueous ammonium chloride solution. Progress of the reaction was monitored by TLC. When the reaction was completed the reaction mixture was filtered 
trough a celite pad and the solid was washed with dichloromethane, the organic layer was separated and the aqueous layer extracted with dichloromethane. The organic layer was dried and the solvent was removed under reduced pressure. The product was purified by column chromatography.

$\left(S_{1}, R_{3 \mathrm{a}}, R_{4}, S_{10}, S_{10 \mathrm{a}}\right)-(+)$-syn-7-exo. It was obtained from syn-6b-exo and purified by column chromatography (pentane-ethyl acetate, 2:1). Yield 77\%. White solid, mp 144-146 ${ }^{\circ} \mathrm{C} .[\alpha]_{\mathrm{D}}{ }^{20}+11.7(c$ 0.24, $\mathrm{CHCl}_{3}$ ). Anal. Calcd. for $\mathrm{C}_{16} \mathrm{H}_{16} \mathrm{O}_{6}: \mathrm{C}, 63.15 ; \mathrm{H}, 5.30$. Found: C, 62.85; H, 5.15. IR (KBr): 1794, 1598, 1173, 1142, 1010. ${ }^{1} \mathrm{H}$ NMR $\delta: 8.02(\mathrm{ddd}, 1 \mathrm{H}, J$ 0.6, 1.4 and 7.7), 7.69 (ddd, $1 \mathrm{H}, J$ 7.8, 7.3 and 1.4), $7.60(\mathrm{ddd}, 1 \mathrm{H}, J 7.7,1.3$ and 0.6), $7.52(\mathrm{ddd}, 1 \mathrm{H}, J$ 7.7, 7.3 and 1.3), $5.64(\mathrm{~d}, 1 \mathrm{H}, J$ 7.0), 5.39 (m, 1H), $4.02(\mathrm{~m}, 1 \mathrm{H}), 3.75(\mathrm{~m}, 1 \mathrm{H}), 3.53(\mathrm{~s}, 3 \mathrm{H}), 3.31(\mathrm{~d}, 1 \mathrm{H}, J$ 9.0), $3.13(\mathrm{ddd}, 1 \mathrm{H}, J$ 9.0, 7.0 and 1.0), $1.34\left(\mathrm{t}, 3 \mathrm{H}, J\right.$ 7.1). ${ }^{13} \mathrm{C}$ NMR $\delta: 193.6,169.2$, and $143.5(\mathrm{C}), 134.9(\mathrm{CH}), 129.6(\mathrm{C}$ and $\mathrm{CH}), 127.5$, $123.6(\mathrm{CH}), 108.1(\mathrm{C}), 101.2,80.3(\mathrm{CH}), 67.3\left(\mathrm{CH}_{2}\right), 54.1(\mathrm{CH}), 54.0\left(\mathrm{CH}_{3}\right), 45.4(\mathrm{CH}), 14.9\left(\mathrm{CH}_{3}\right)$.

$\left(S_{1}, S_{3 \mathrm{a}}, S_{4}, R_{10}, R_{10 \mathrm{a}}\right)-(+)$-anti-7-exo. It was obtained from anti-6b-exo. It was purified by column chromatography (eluent pentane-ethyl acetate, 3:1). Quantitative yield. $[\alpha]_{\mathrm{D}}{ }^{20}+293.4\left(c 0.21, \mathrm{CHCl}_{3}\right)$, mp 210-212 ${ }^{\circ} \mathrm{C} .{ }^{1} \mathrm{H}$ NMR $\delta: 8.03(\mathrm{ddd}, 1 \mathrm{H}, J$ 7.7, 1.4 and 0.6), 7.71 (ddd, $1 \mathrm{H}, J$ 7.8, 7.3 and 1.4), 7.59 (ddd, 1H, J 7.8, 1.3 and 0.6), $7.54(\mathrm{ddd}, 1 \mathrm{H}, J$ 7.7, 7.3, and 1.3), $5.53(\mathrm{~d}, 1 \mathrm{H}, J 1.7), 4.90(\mathrm{~m}, 1 \mathrm{H})$, 3.91(m, 1H), $3.64(\mathrm{~m}, 1 \mathrm{H}), 3.52(\mathrm{~s}, 3 \mathrm{H}), 3.37(\mathrm{dd}, 1 \mathrm{H}, J 8.2$ and 0.6$), 2.94(\mathrm{ddd}, 1 \mathrm{H}, J 8.2,1.7$ and 1.0), $1.22(\mathrm{t}, 3 \mathrm{H}, \mathrm{J} 7.0) .{ }^{13} \mathrm{C}$ NMR $\delta: 192.0,170.5$ and $143.2(\mathrm{C}), 135.1,129.8(\mathrm{CH}), 129.3(\mathrm{C}) 127.7,123.6$ $(\mathrm{CH}), 108.1(\mathrm{C}), 106.8$ and $84.5(\mathrm{CH}), 65.7\left(\mathrm{CH}_{2}\right), 54.1\left(\mathrm{CH}_{3}\right), 53.5$ and $49.2(\mathrm{CH}), 14.8\left(\mathrm{CH}_{3}\right) . \mathrm{HRMS}$ Calcd for $\mathrm{C}_{16} \mathrm{H}_{17} \mathrm{O}_{6}[\mathrm{M}+\mathrm{H}]$ 305.1025, found 305.1024.

$\left(R_{1}, R_{3 \mathrm{a}}, R_{4}, S_{10}, S_{10 \mathrm{a}}\right)$-(-)-anti-7-exo. It was obtained from anti-6a-exo and it was purified by column chromatography (eluent pentane-ethyl acetate, 3:1). Yield 74\%. $[\alpha]_{\mathrm{D}}{ }^{20}-291.0\left(c 0.13, \mathrm{CHCl}_{3}\right)$. White solid, mp 206-208 ${ }^{\circ} \mathrm{C}$. Anal. Calcd. for $\mathrm{C}_{16} \mathrm{H}_{16} \mathrm{O}_{6}$ : C, 63.15; H, 5.30. Found: C, 62.77; H, 5.28. IR (KBr): $1775,1700,1600,1169,1116,1070$.

$\left(S_{1}, S_{3 \mathrm{a}}, R_{4}, S_{10}, R_{10 \mathrm{a}}\right)$-(-)-anti-7-endo. It was obtained from anti-6b-endo and purified by column chromatography (eluent pentane-ethyl acetate, 6:1). Yield. 74\%. $[\alpha]_{\mathrm{D}}{ }^{20}-107.6\left(c 0.37, \mathrm{CHCl}_{3}\right)$. White 
solid, mp 104-106 ${ }^{\circ} \mathrm{C}$. Anal. Calcd. for $\mathrm{C}_{16} \mathrm{H}_{16} \mathrm{O}_{6}: \mathrm{C}, 63.15 ; \mathrm{H}, 5.30$. Found: $\mathrm{C}, 62.86 ; \mathrm{H}, 5.37$. IR (KBr): 1773, 1709, 1600, 1323, 1302, 1121, 1051, 943. ${ }^{1} \mathrm{H}$ NMR $\delta: 8.00(\mathrm{dd}, 1 \mathrm{H}, J 7.8$ and 1.3), $7.68(\mathrm{~m}, 1 \mathrm{H})$, $7.59(\mathrm{~m}, 1 \mathrm{H}), 7.51(\mathrm{~m}, 1 \mathrm{H}), 5.04(\mathrm{~d}, 1 \mathrm{H}, J 1.3), 5.01(\mathrm{~d}, 1 \mathrm{H}, J 8.9), 3.82(\mathrm{~d}, 1 \mathrm{H}, J 10.6), 3.70(\mathrm{~m}, 1 \mathrm{H})$, $3.65(\mathrm{~m}, 1 \mathrm{H}), 3.62(\mathrm{~s}, 3 \mathrm{H}), 3.47(\mathrm{~m}, 1 \mathrm{H}), 1.16(\mathrm{t}, 3 \mathrm{H}, \mathrm{J} 7.0) .{ }^{13} \mathrm{C}$ NMR $\delta: 192.7,172.3,141.0(\mathrm{C}), 134.8$ $(\mathrm{CH}), 130.0(\mathrm{C}), 129.8,126.7$ and $125.7(\mathrm{CH}), 108.1(\mathrm{C}), 101.6$ and $81.4(\mathrm{CH}), 65.5\left(\mathrm{CH}_{2}\right), 52.9(\mathrm{CH})$, $52.4\left(\mathrm{CH}_{3}\right), 48.6(\mathrm{CH}), 14.7\left(\mathrm{CH}_{3}\right)$.

$\left(R_{1}, R_{3 \mathrm{a}}, S_{4}, R_{10}, S_{10 \mathrm{a}}\right)$-(+)-anti-7-endo. It was obtained from anti-6a-endo and purified by column chromatography (eluent pentane-ethyl acetate, 6:1). Yield 76\%. $[\alpha]_{\mathrm{D}}{ }^{20}+105.0\left(c 0.31, \mathrm{CHCl}_{3}\right)$. White solid, mp 108-110 ${ }^{\circ} \mathrm{C}$. Anal. Calcd. for $\mathrm{C}_{16} \mathrm{H}_{16} \mathrm{O}_{6}: \mathrm{C}, 63.15 ; \mathrm{H}, 5.30$. Found: $\mathrm{C}, 62.97 ; \mathrm{H}, 5.34$. 


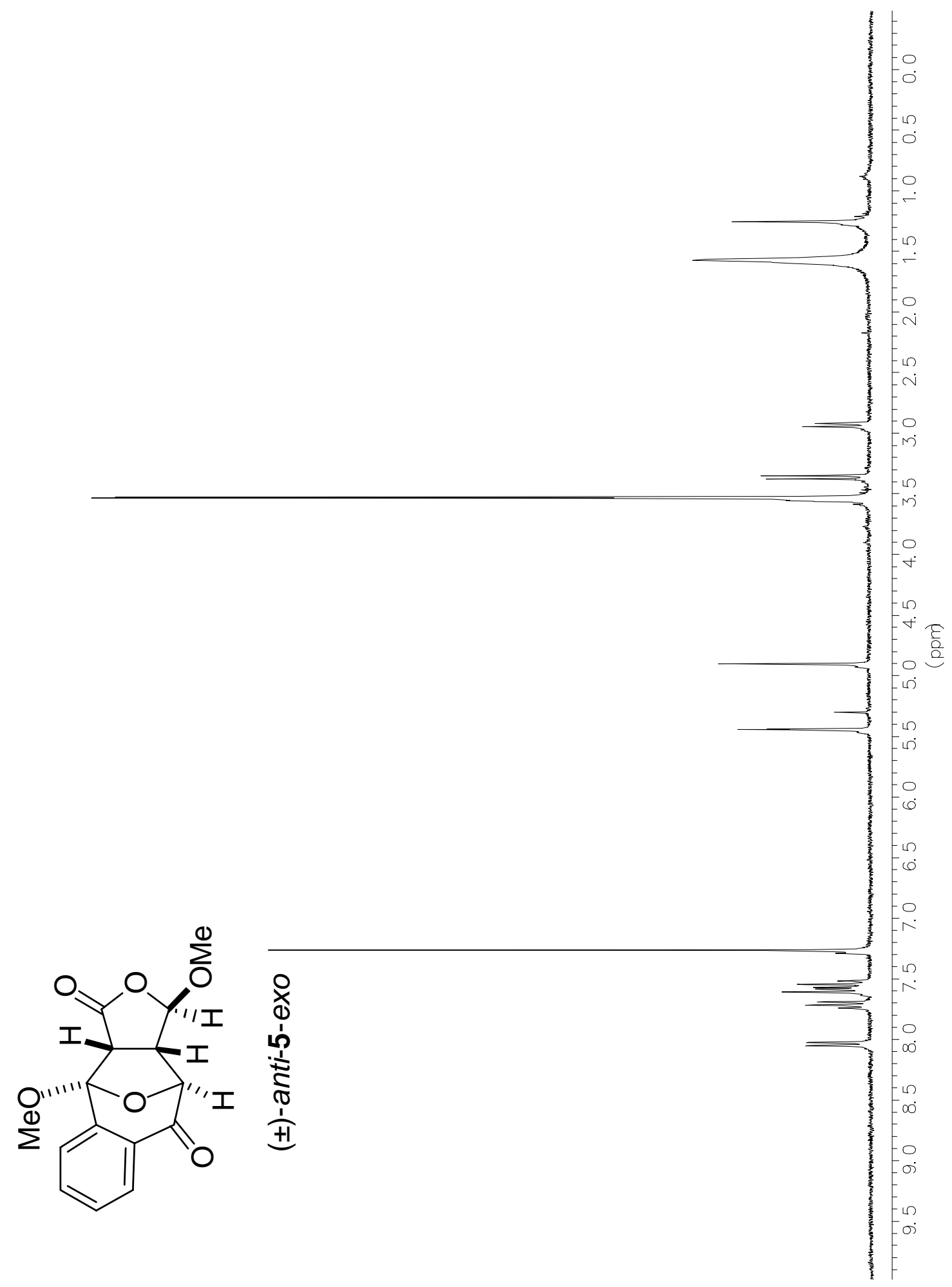




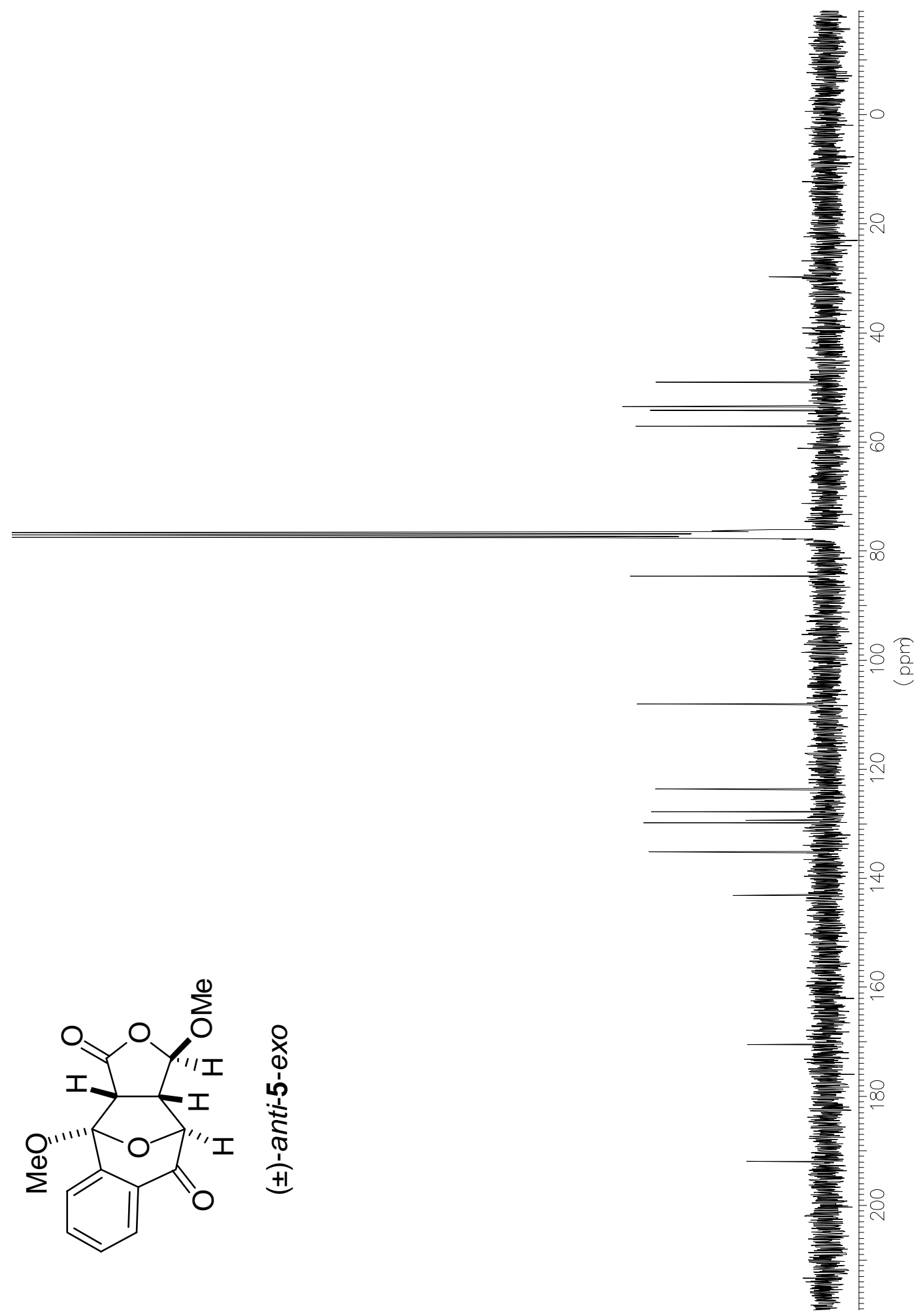




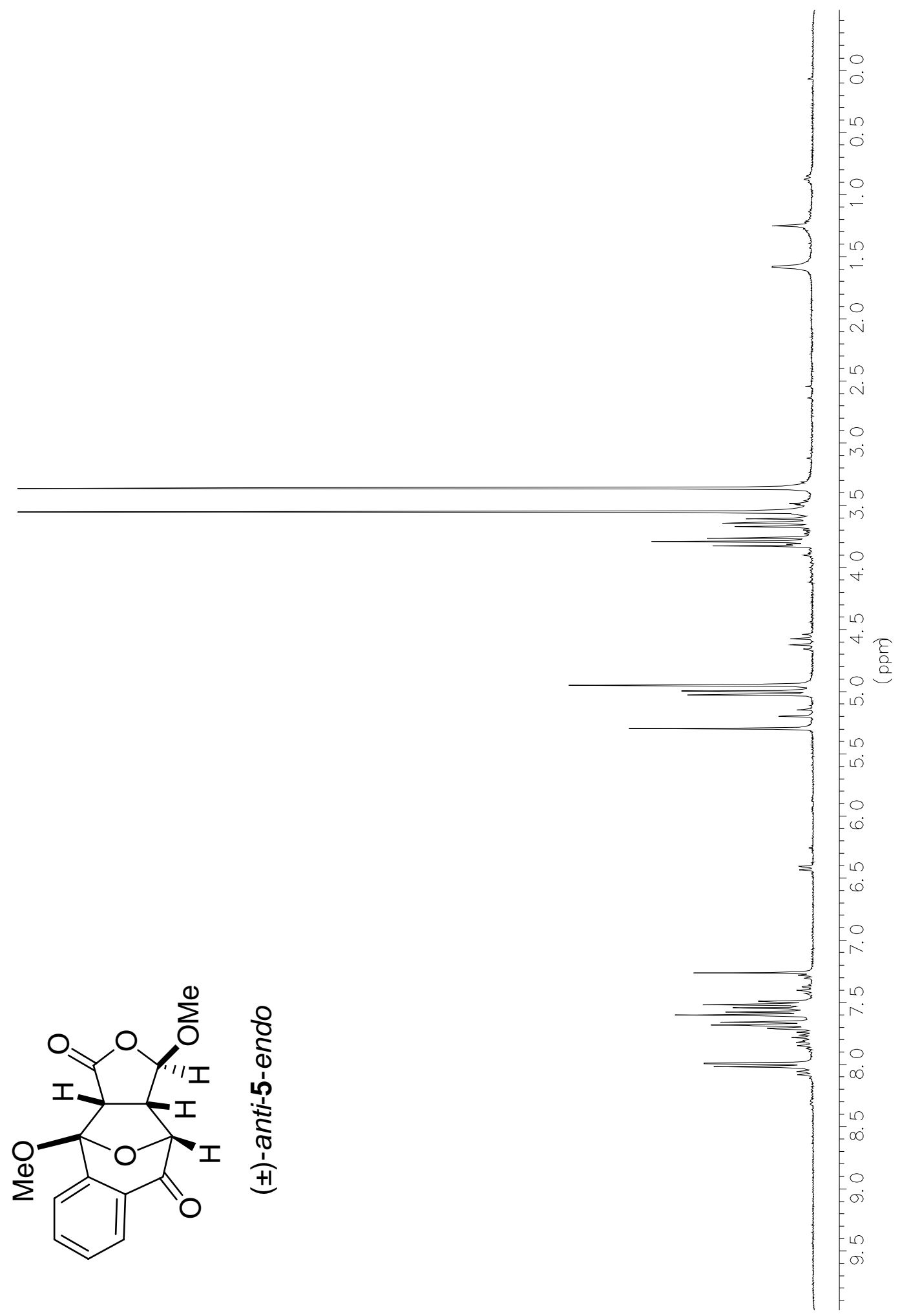




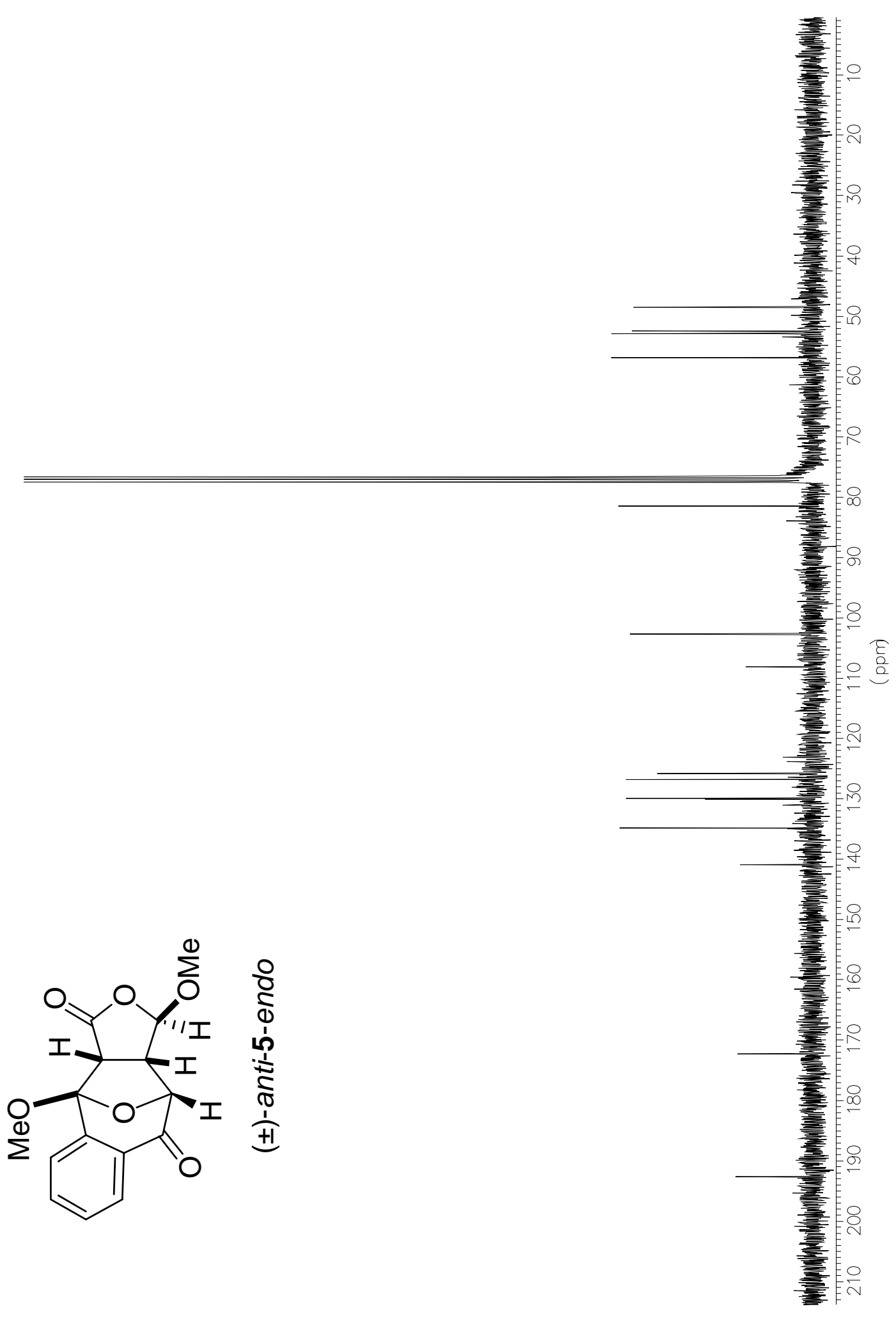




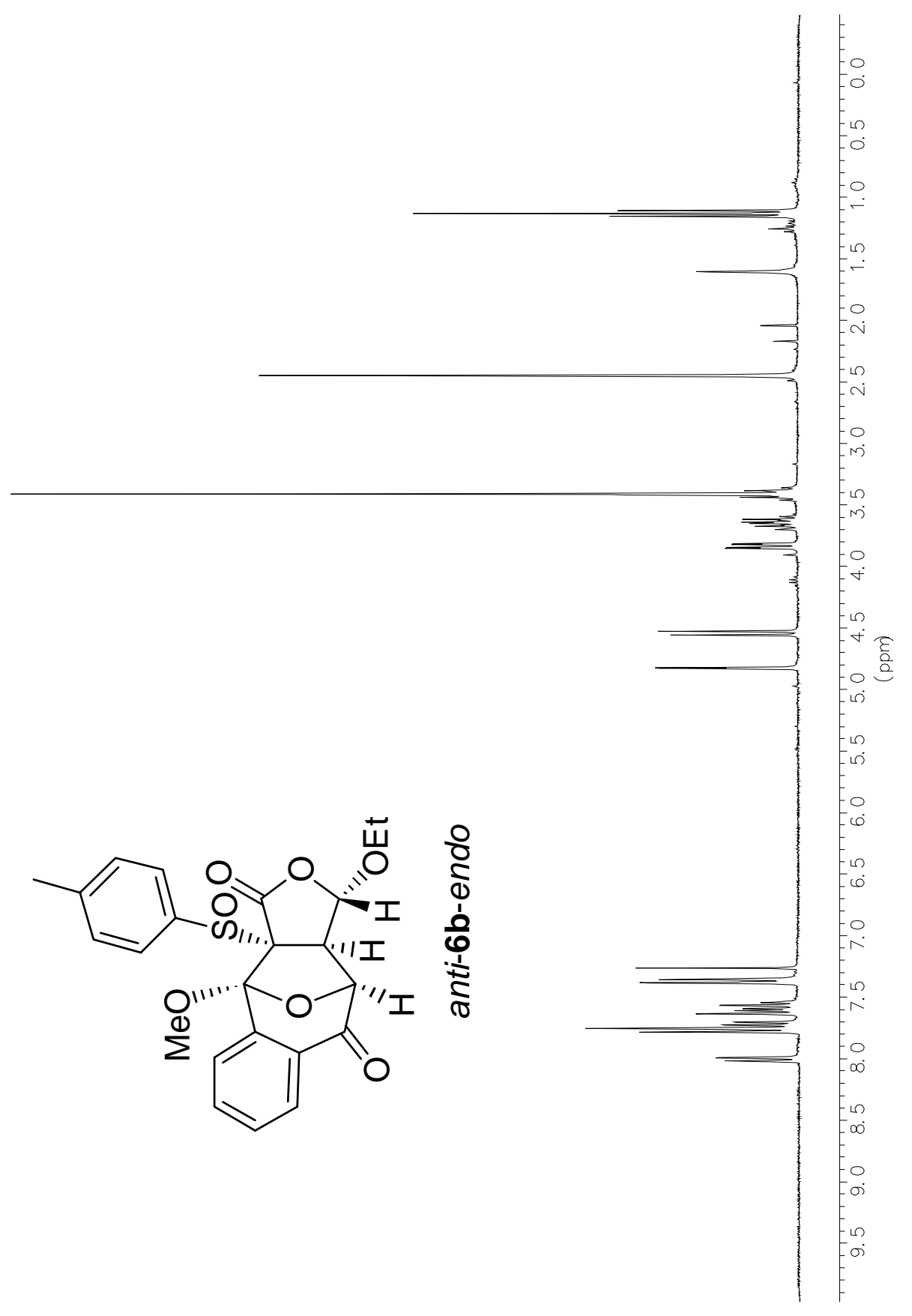




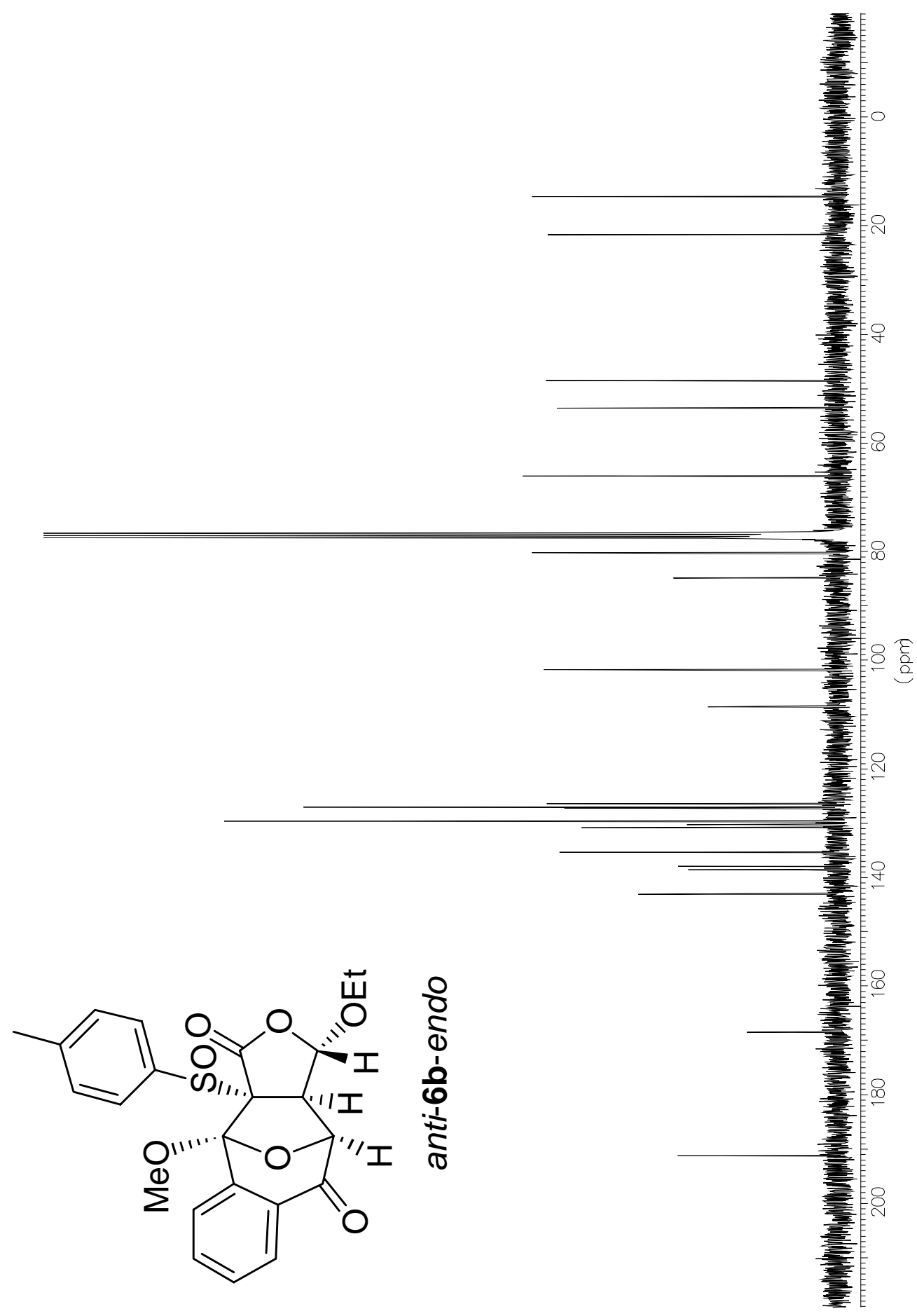



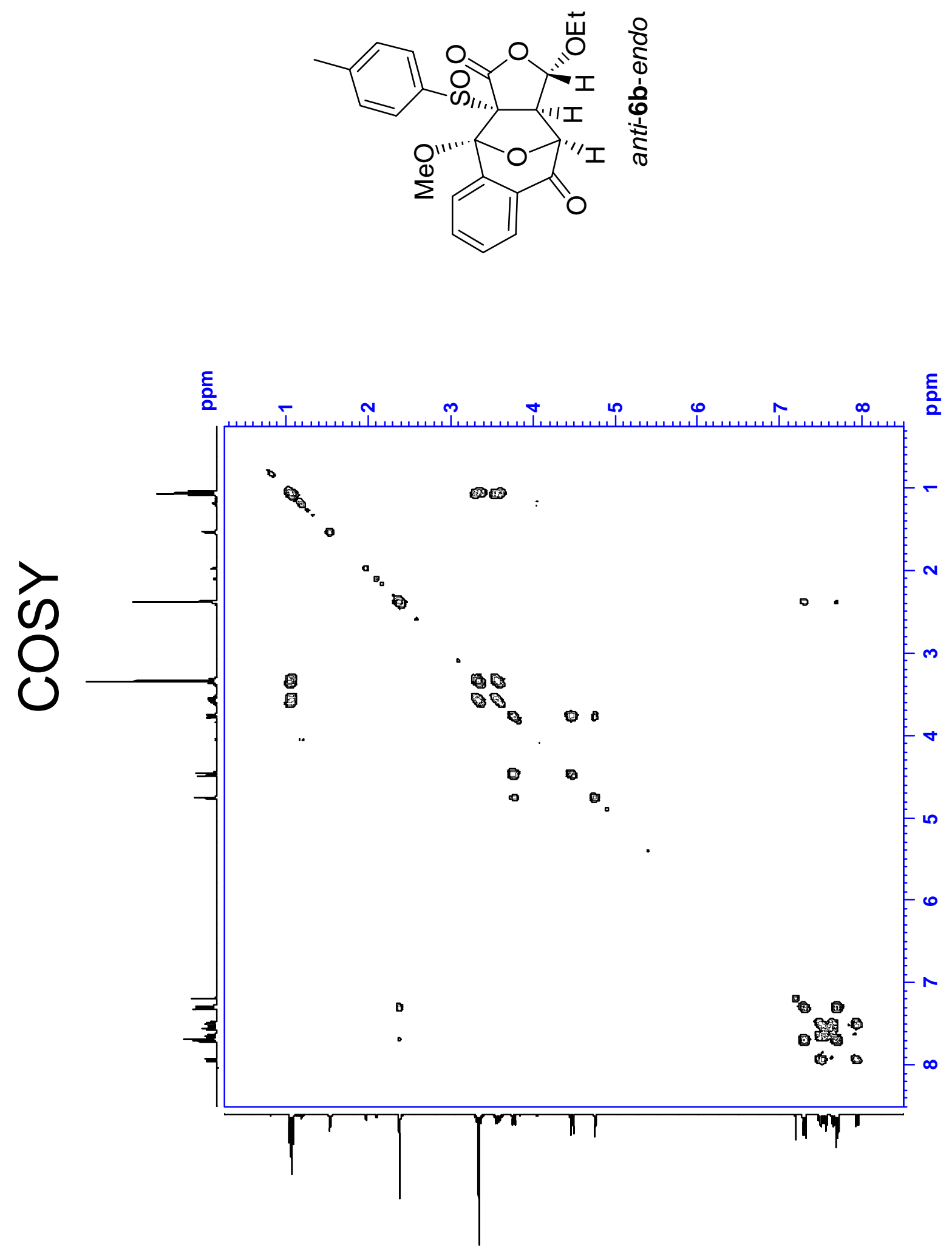

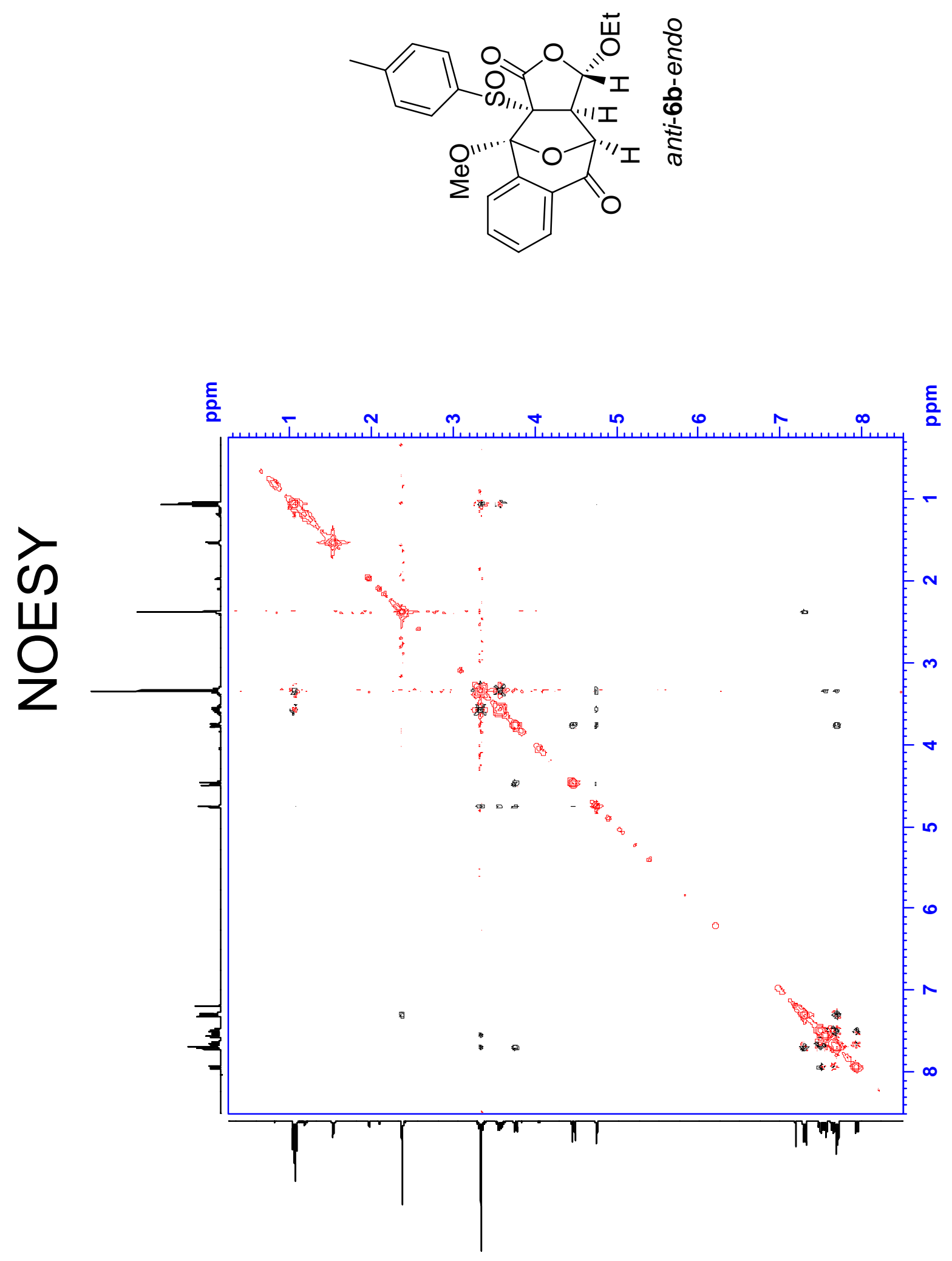

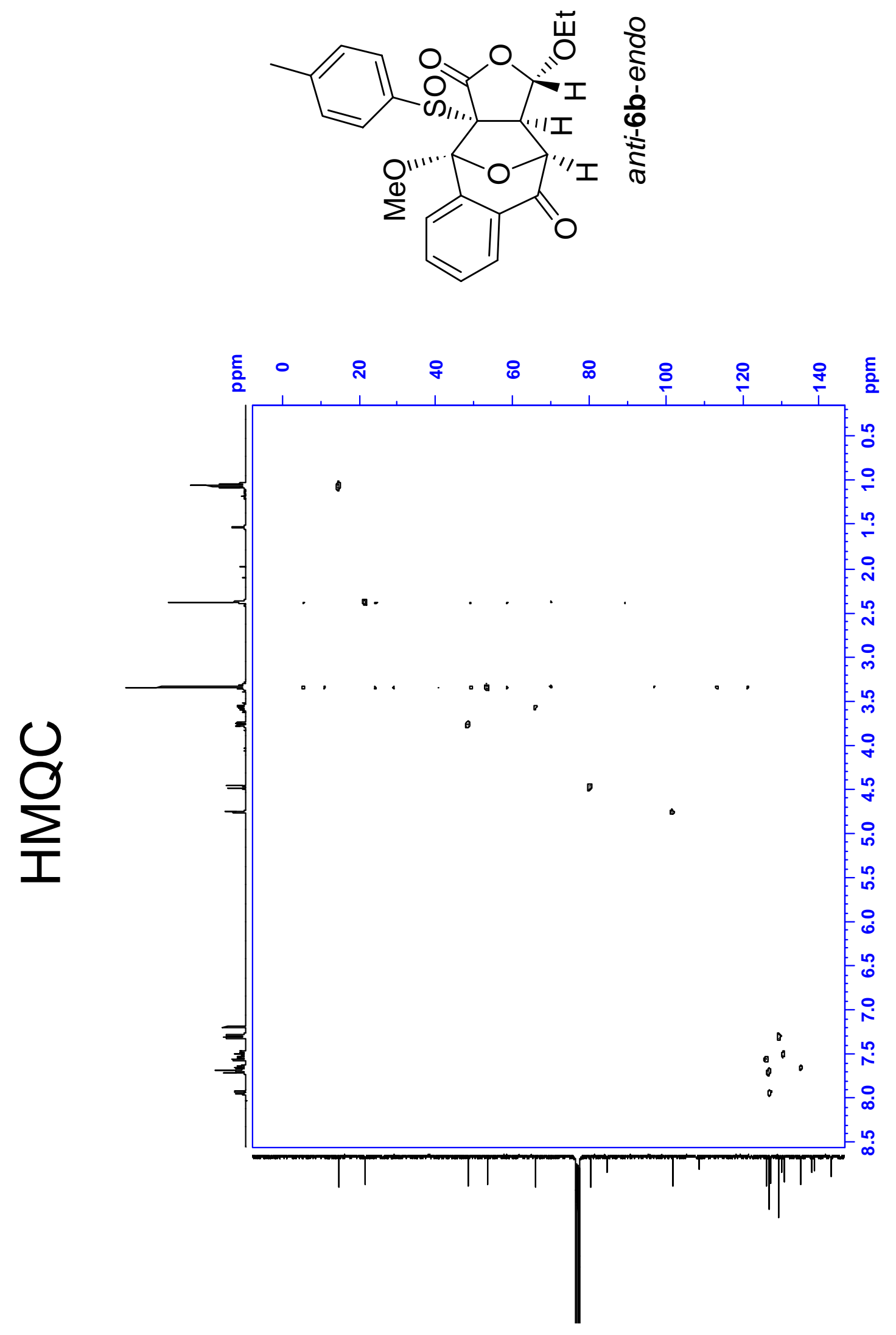


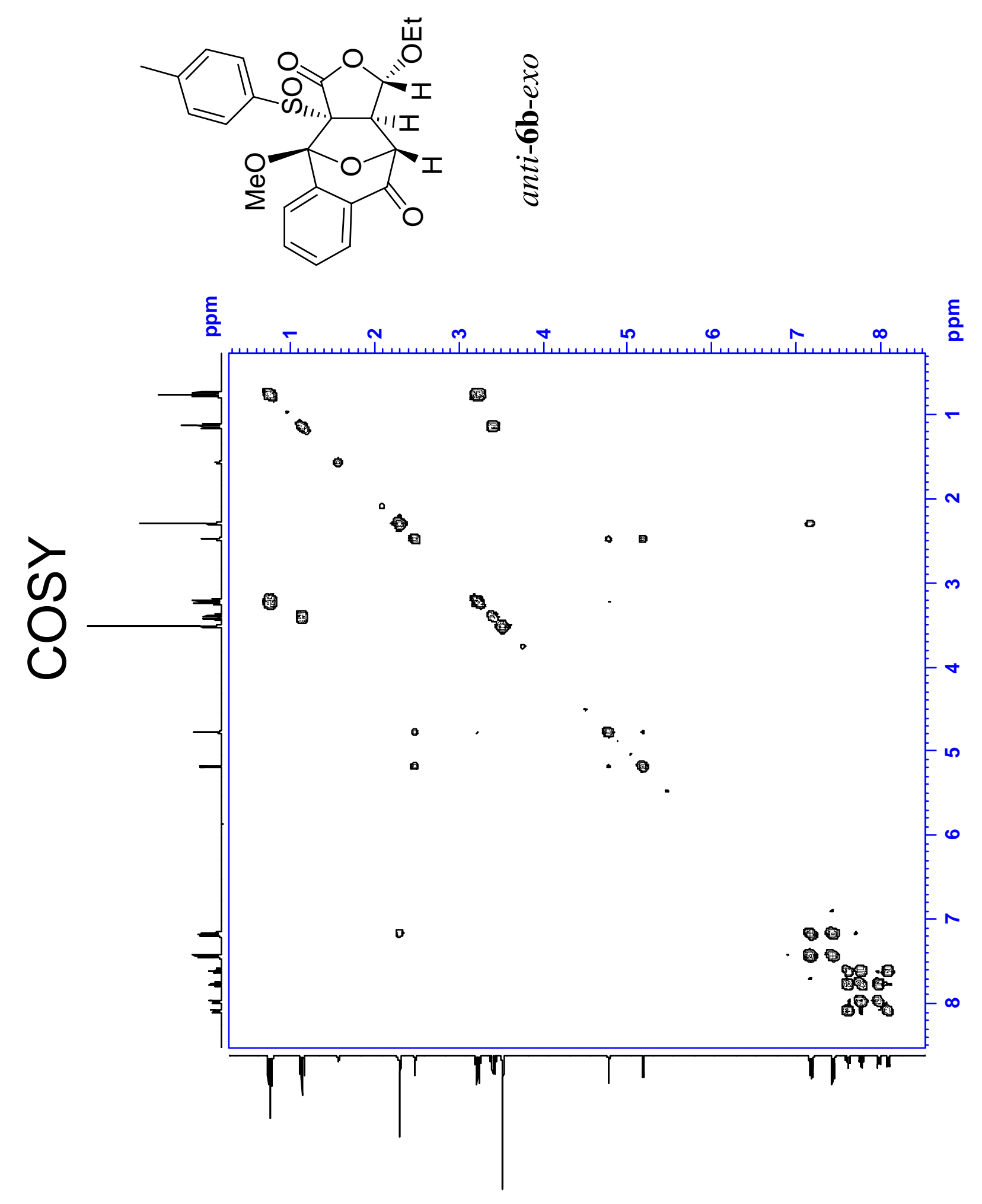



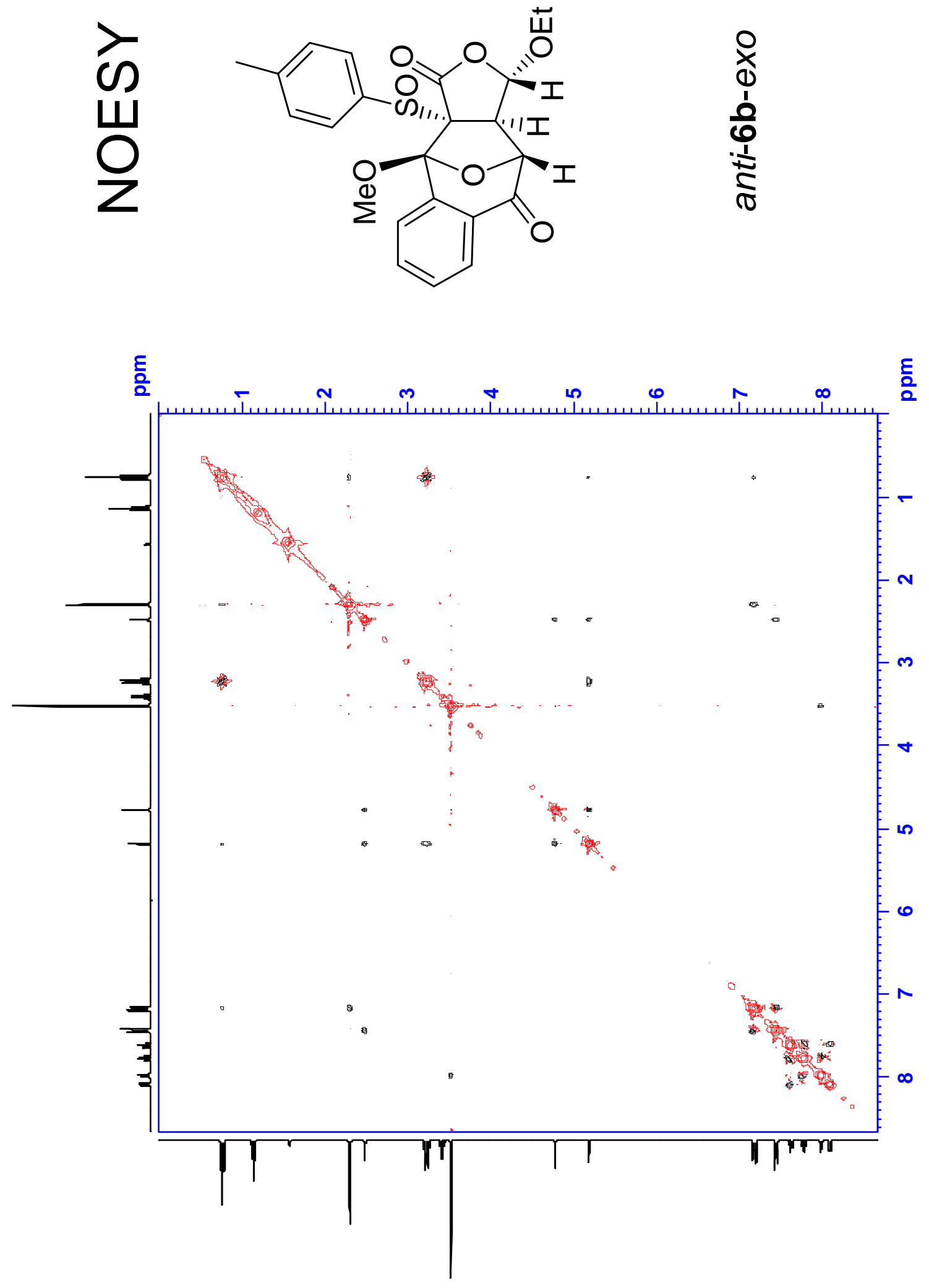


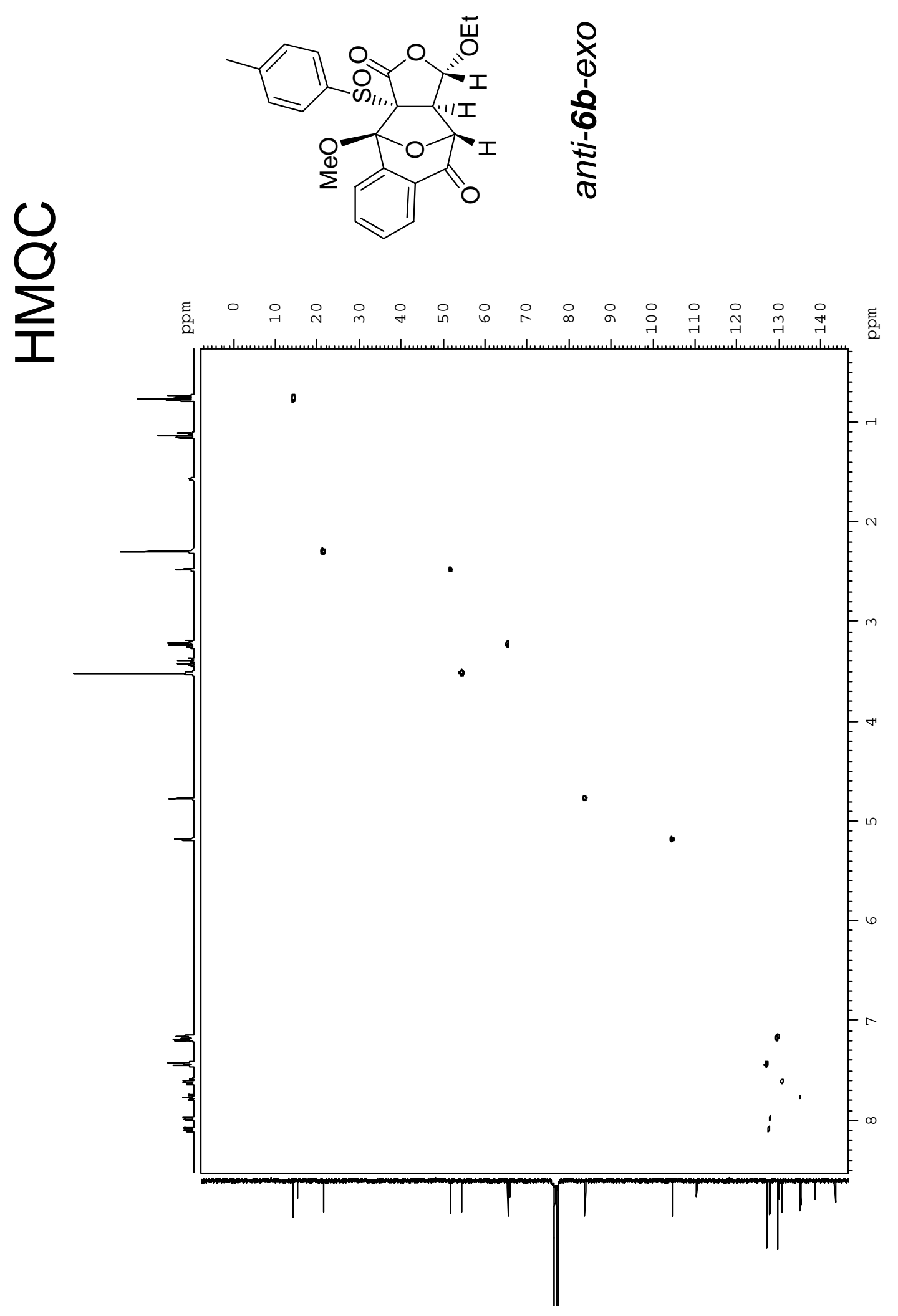



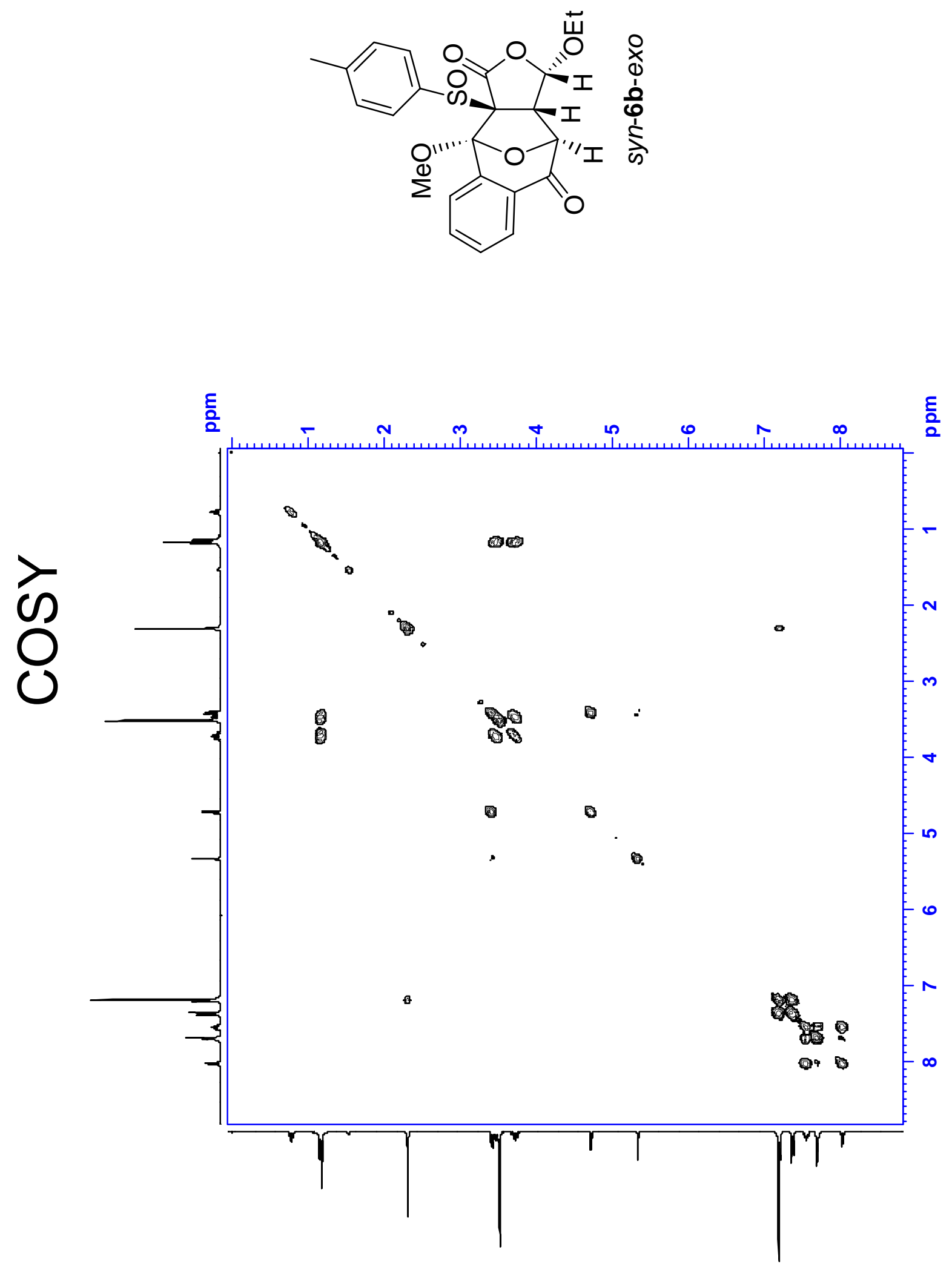

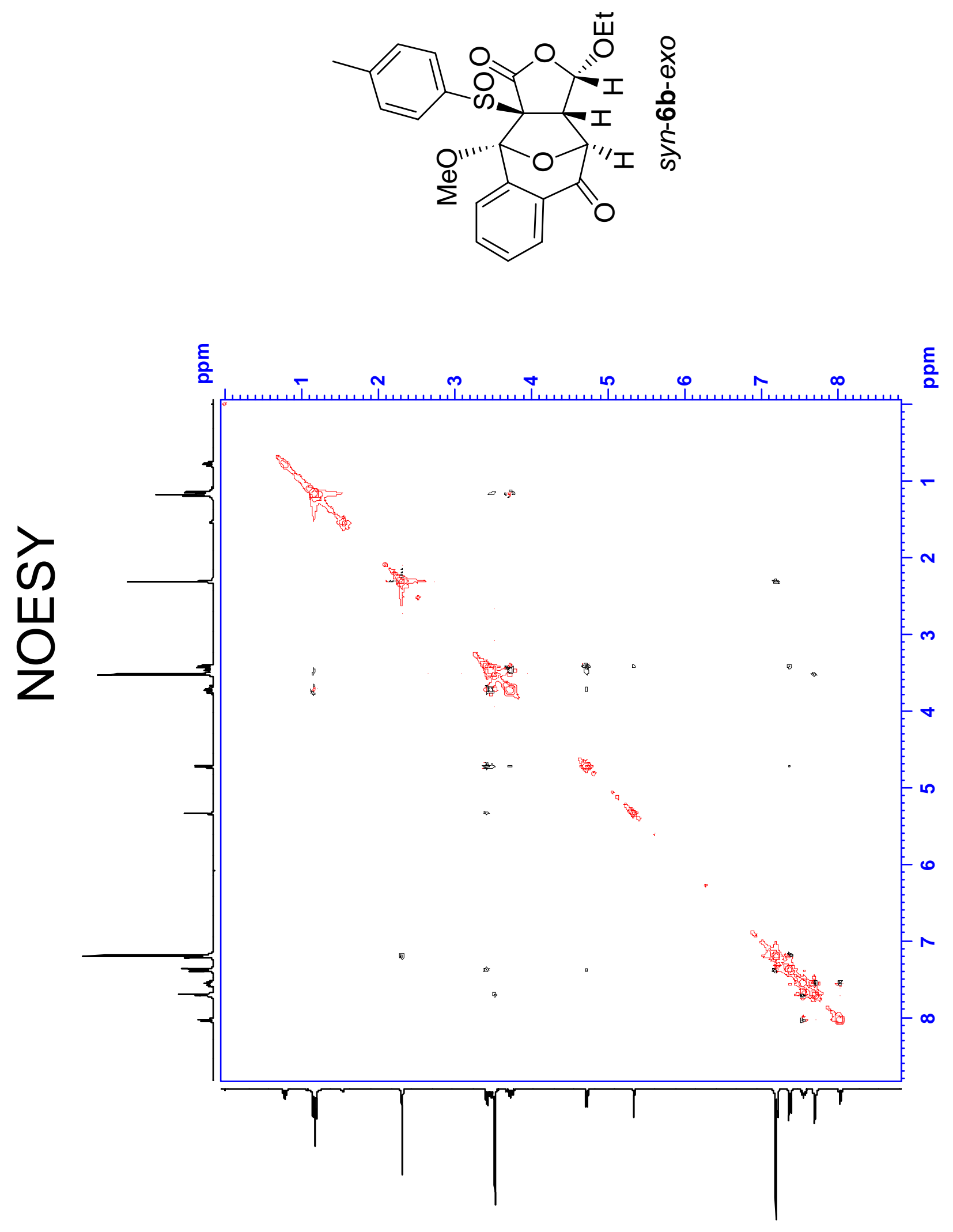


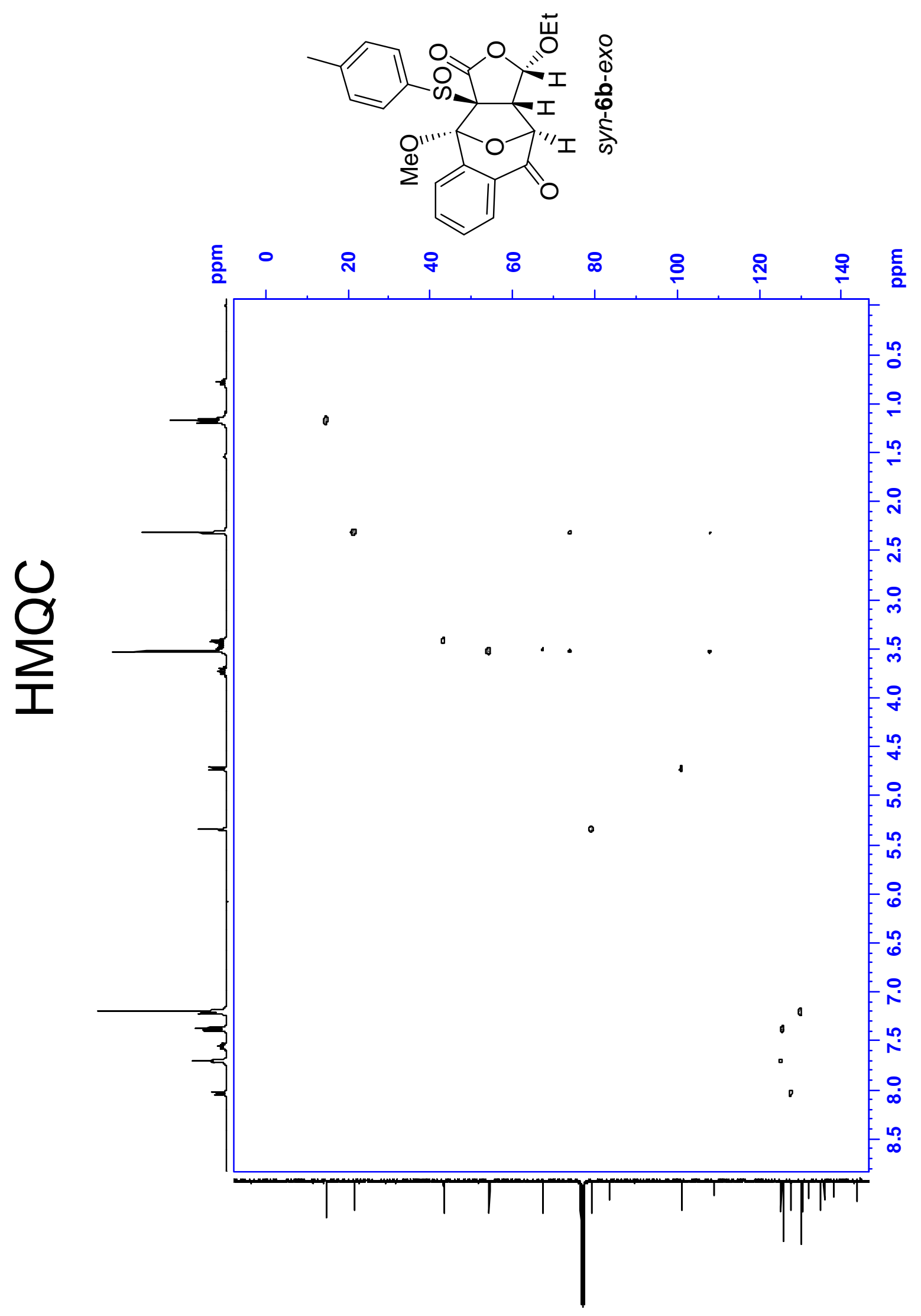




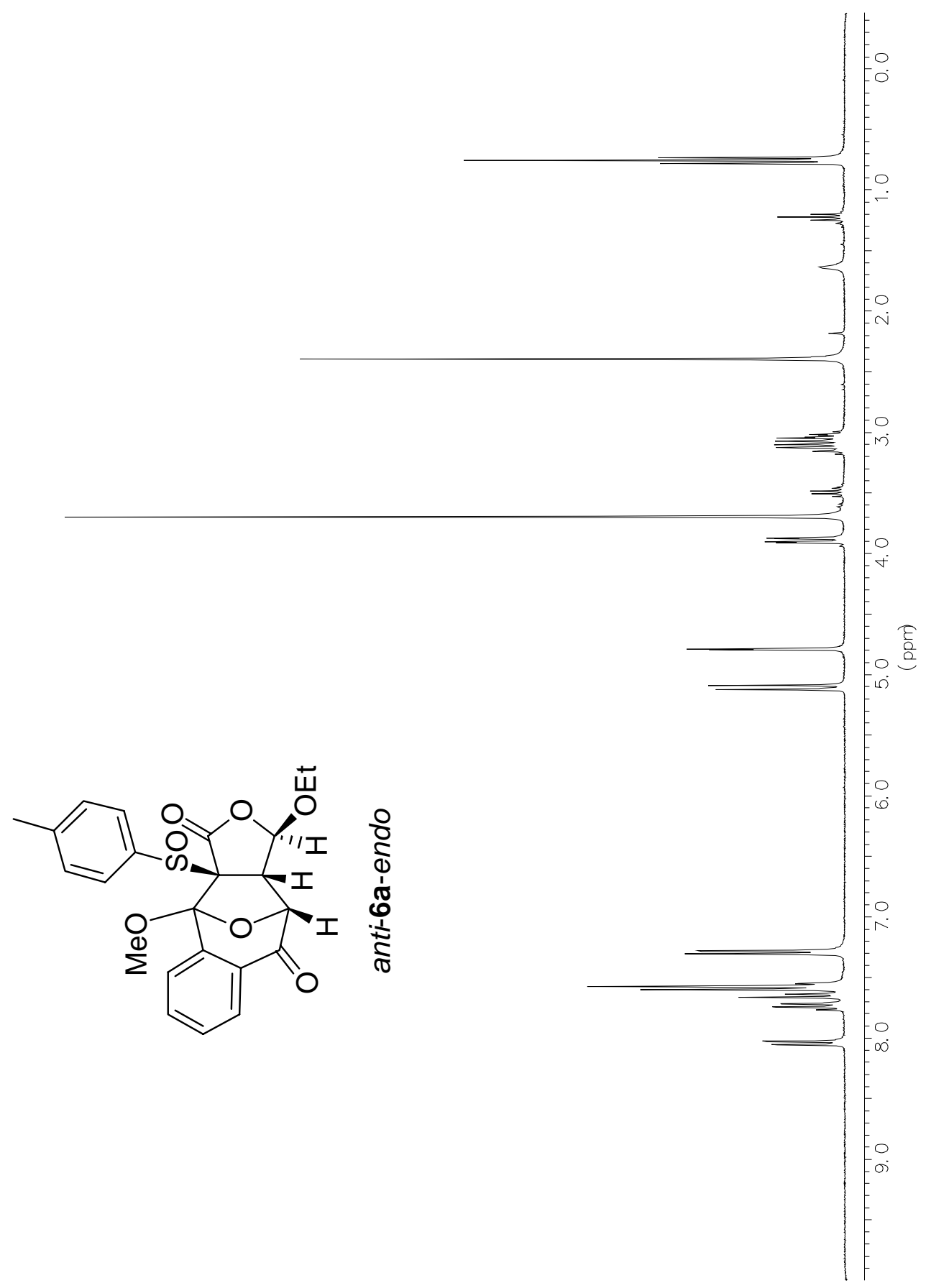




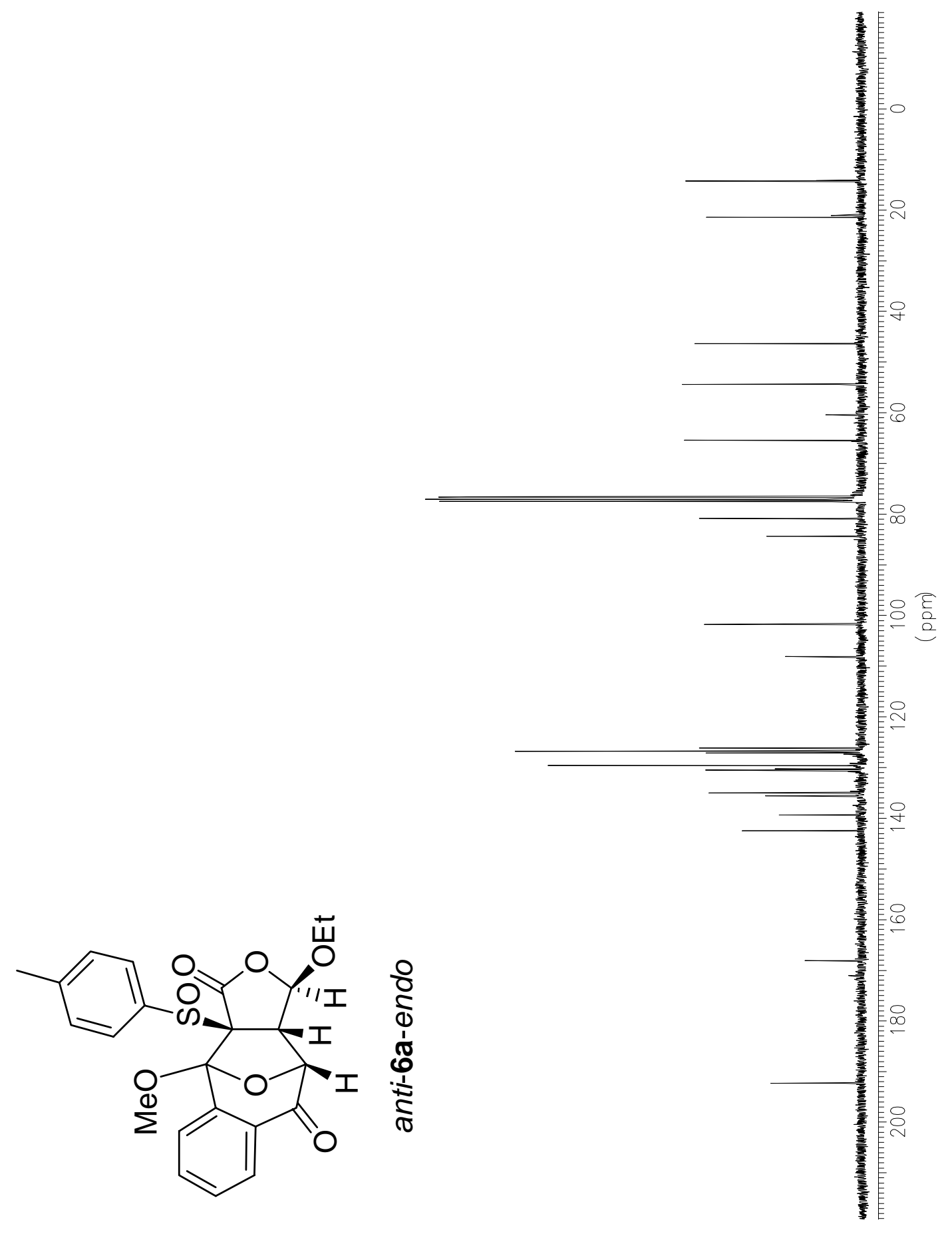




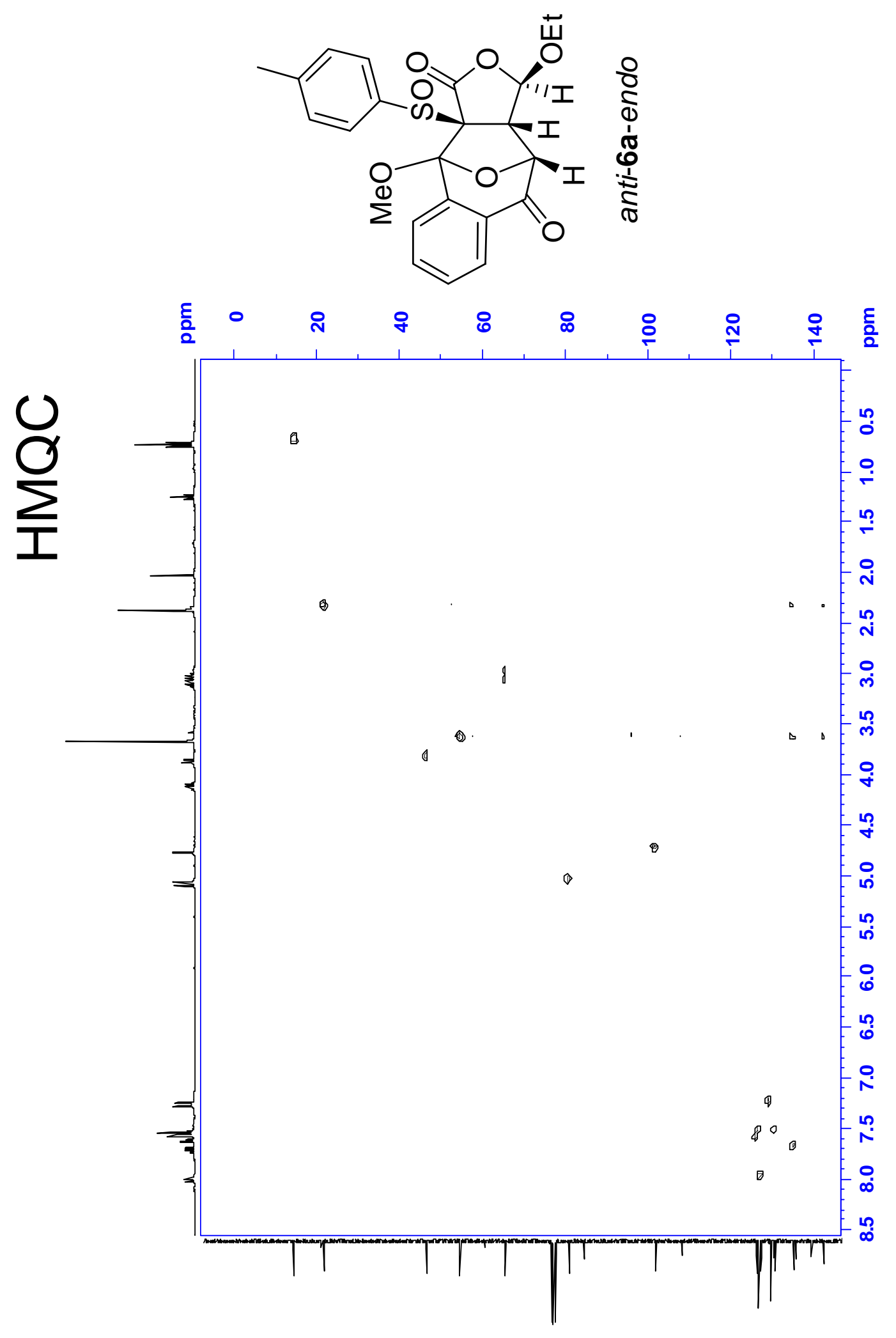



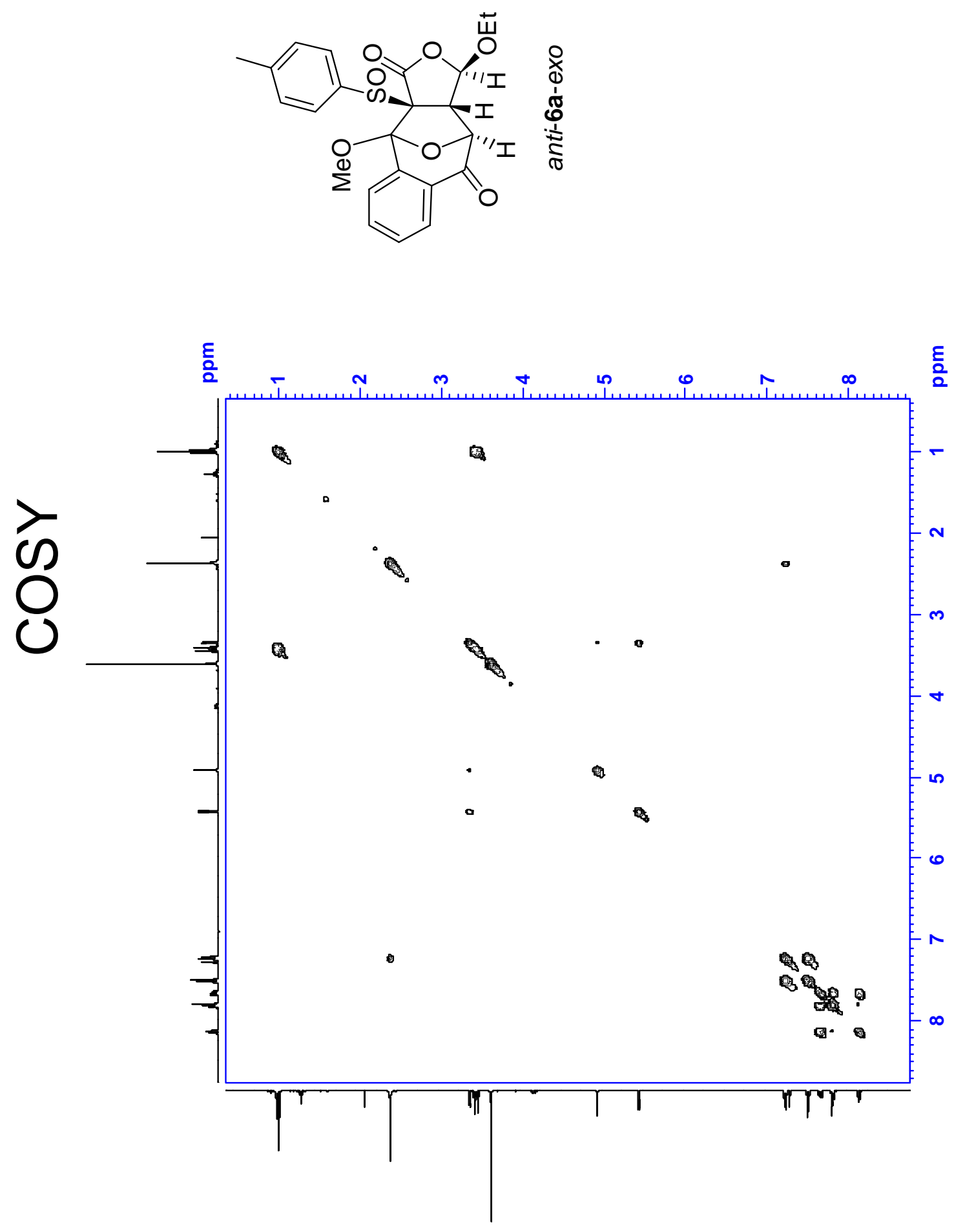

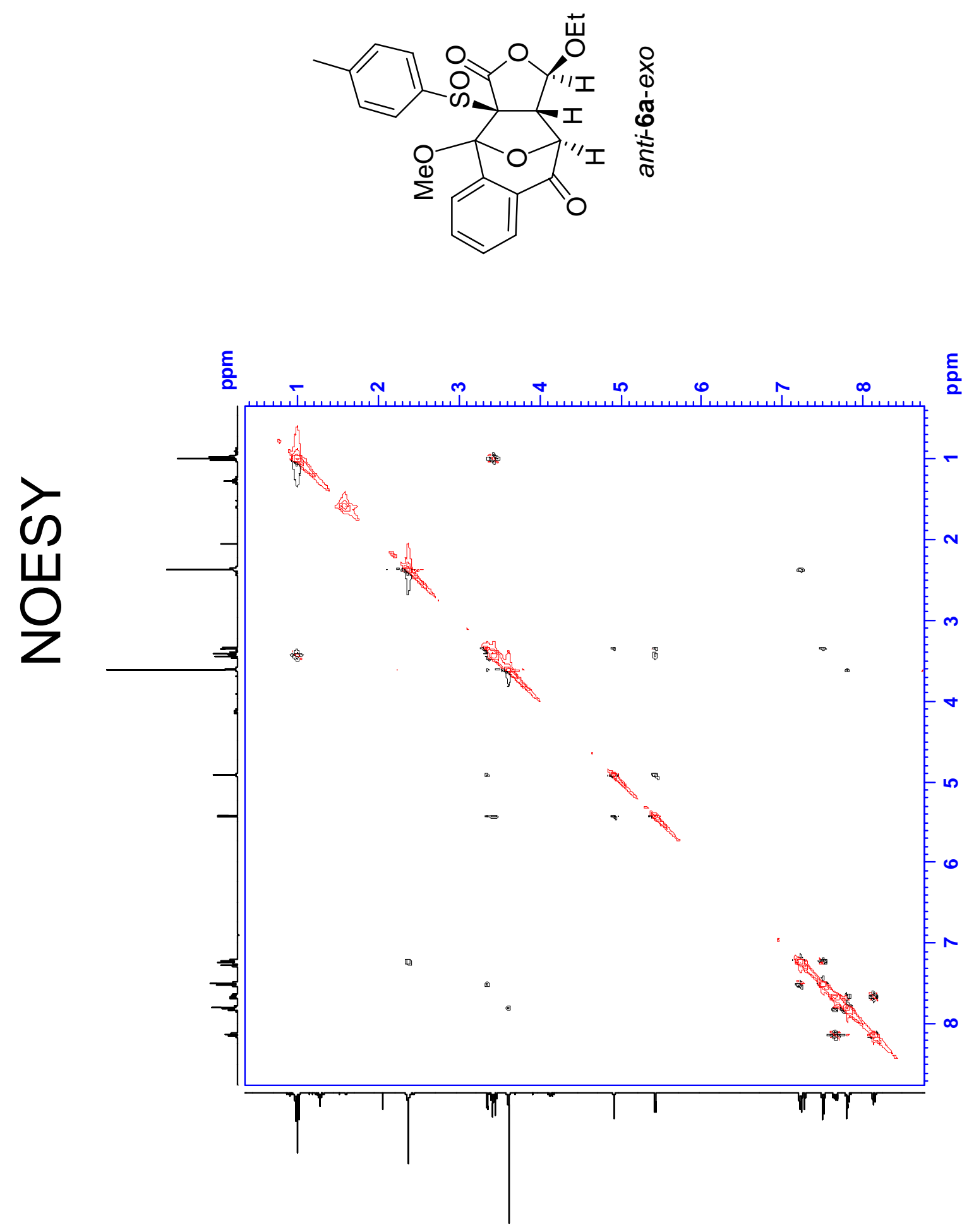


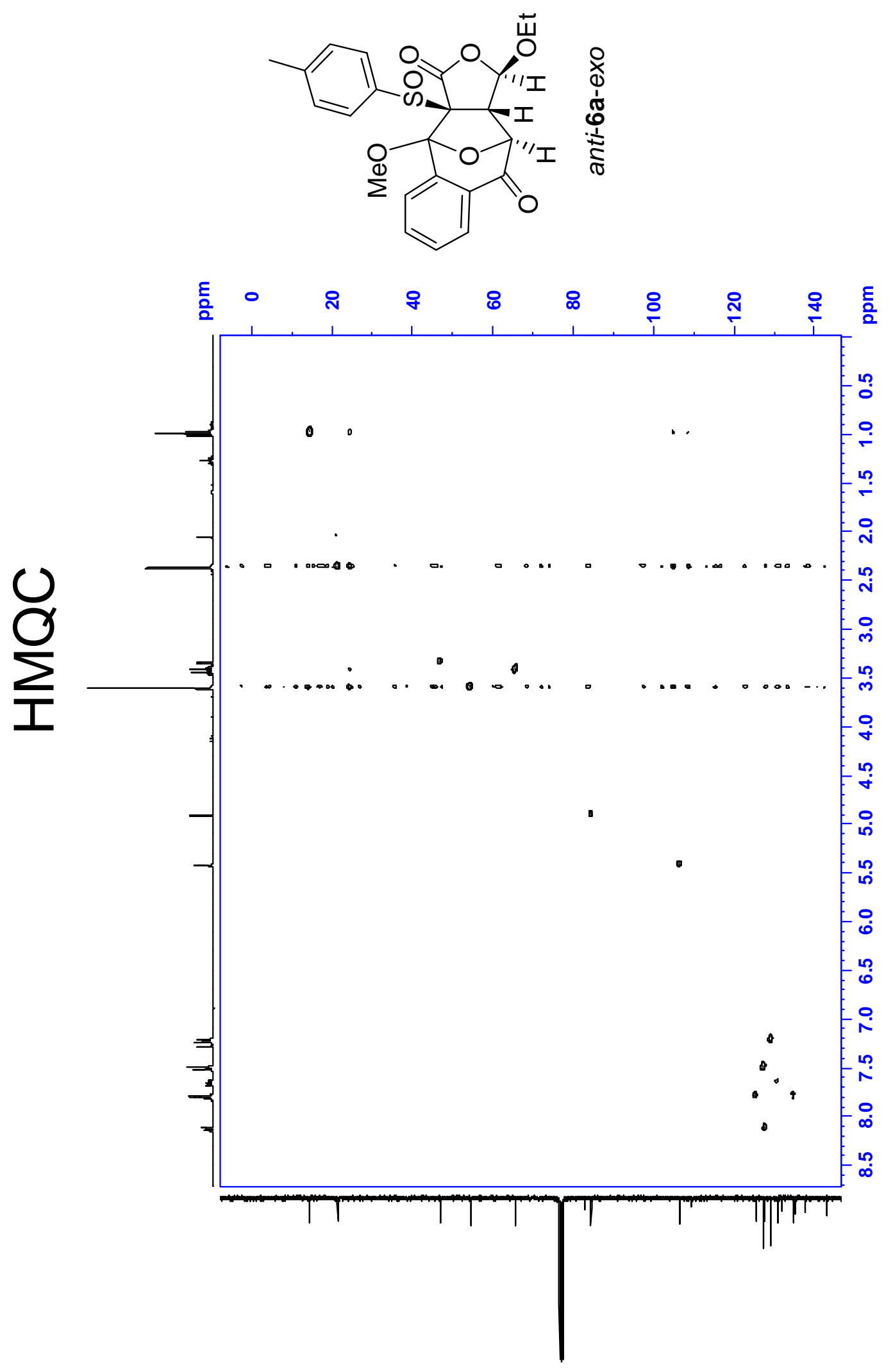




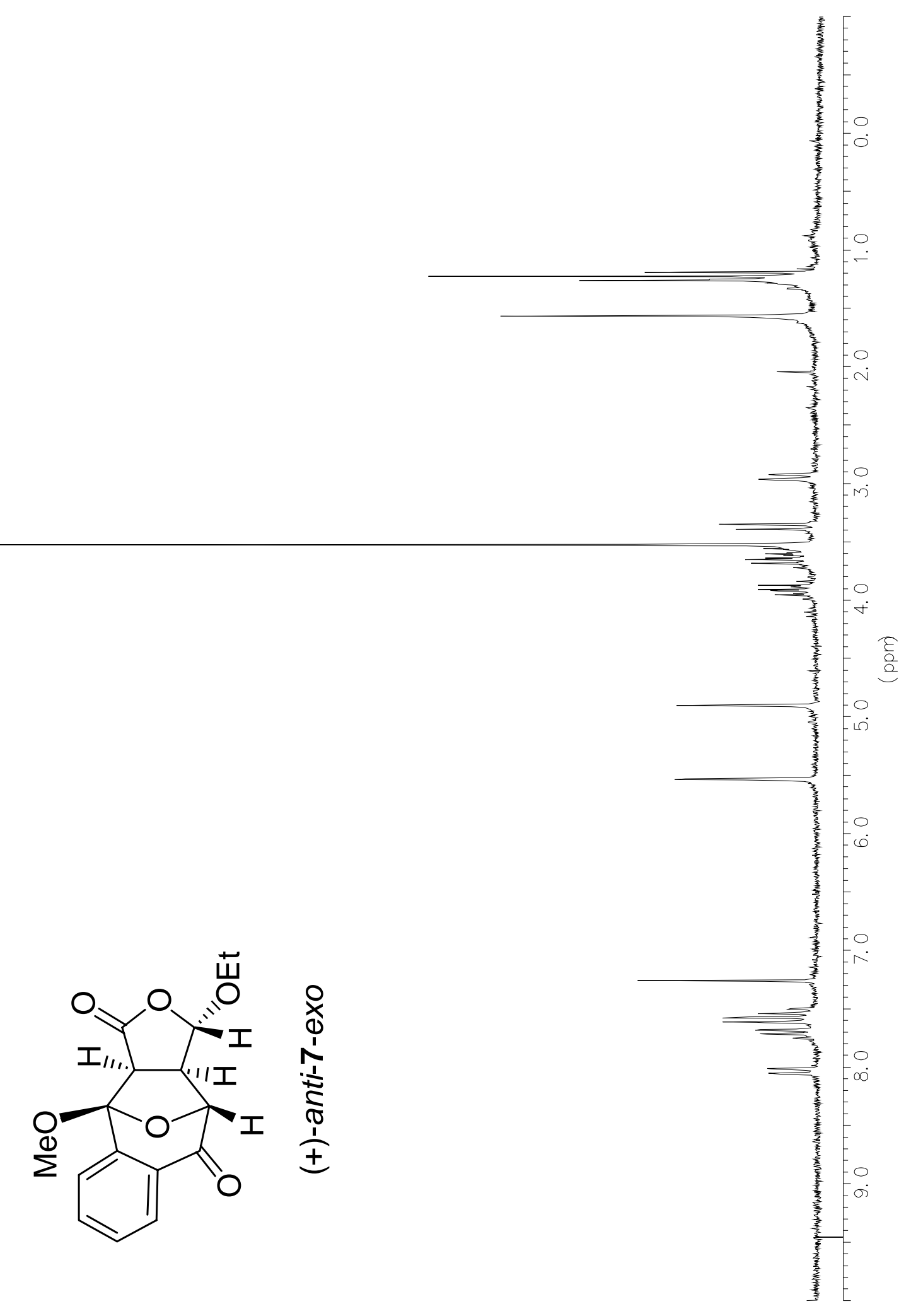



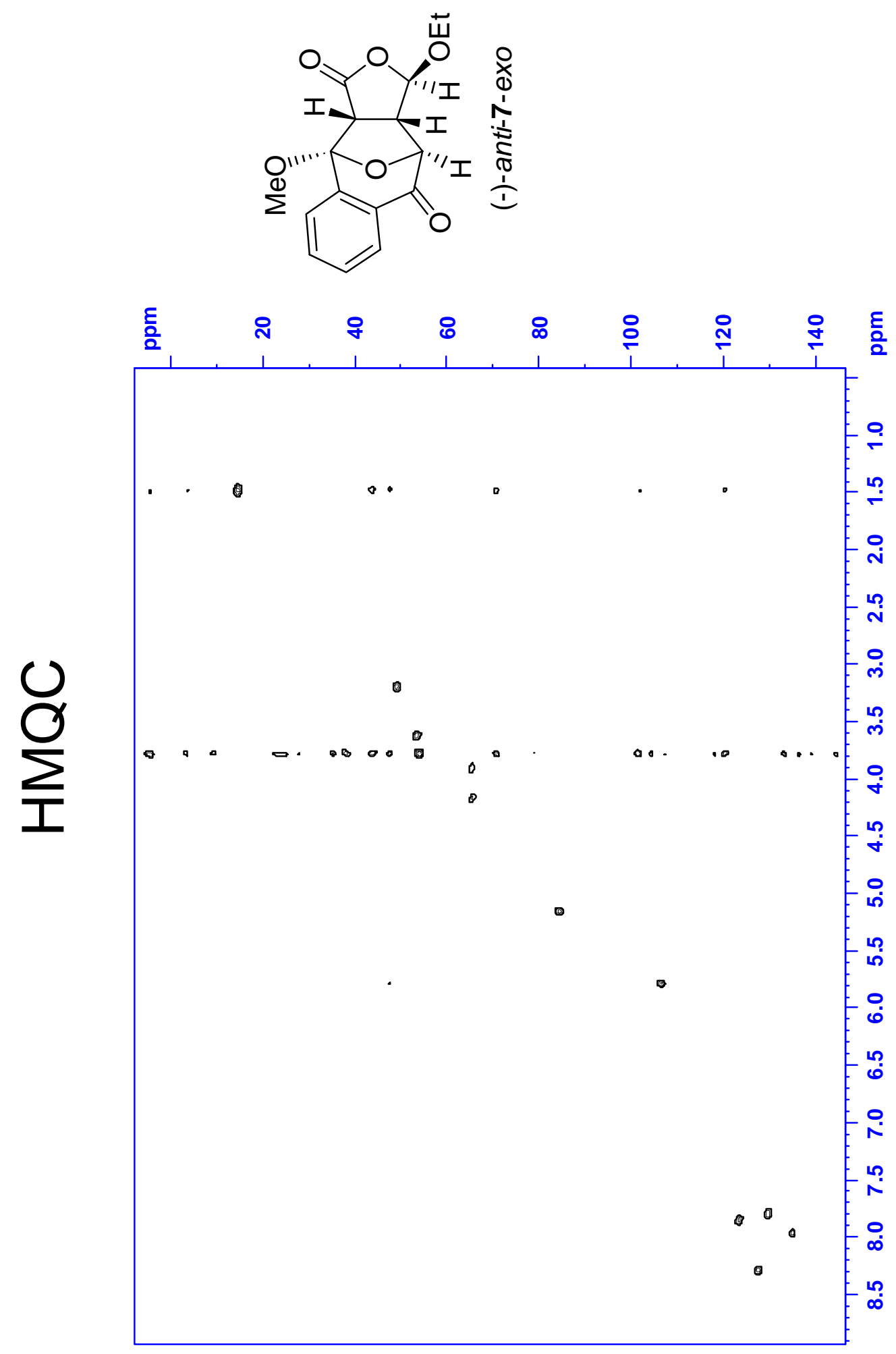